Bull. Austral. Math. Soc.

VOL. 38 (1988) [19-21]

\title{
QUASI-ADDITIVE SOLUTIONS OF NONLINEAR DIFFERENTIAL EQUATIONS II
}

\begin{abstract}
A.S.JONES
In a previous paper, the author sought to classify those solutions of second order nonlinear ordinary differential equations which can be expressed as sums of solutions of related equations. In that paper one sub-class of solutions was overlooked. This paper is to remedy that defect.
\end{abstract}

\section{INTRODUCTION}

In a recent paper [1] the author showed that solutions of differential equations of the form $\ddot{y}=p(y)$, where $p$ is a polynomial of degree two or three, can be represented as infinite sums of solutions of related equations. The second part of the paper sought to classify all real solutions of equations of this type. This classification was, unfortunately, defective in the case where $p$ is a cubic polynomial and the solution has a non-vanishing derivative. It was incorrectly assumed that in order for the solutions to be real on the real axis, it was necessary for the poles of the elliptic functions which are the solutions to be symmetrically placed with respect to both the real and imaginary axes. In this paper it is shown that this condition places restrictions on the form of the polynomial $p$ and therefore excludes one class of solutions. Fortunately only a slight modification is required to correct the omission.

\section{ELLIPTIC FUNCTIONS WITH SYMMETRIC POLES}

The class of solutions under discussion was formed by taking doubly infinite sums of functions of the form

$$
g(t)=t^{-1}-(t+\omega(1+i \lambda) / 2)^{-1}
$$

with periods $\omega$ (real) and $i \lambda \omega$. These sums give rise to elliptic functions with poles arranged at the vertices $(0, \omega(1+i \lambda) / 2, \omega, \omega(1-i \lambda) / 2)$ of a parallelogram. Hence, with the exception of the pole at $t=0$, we can arrange the poles in pairs at $a,-a$ having the same residue. Since $1 /(t-a)+1 /(t+a)=2 t /\left(t^{2}-a^{2}\right)$, this shows that the solution function is odd in $t$, and therefore satisfies a differential equation of the form

$$
\ddot{y}=2 y^{3}+a_{1} y
$$

Received 3 September 1987

Copyright Clearance Centre, Inc. Serial-fee code: 0004-9729/88 \$A2.00+0.00. 
Furthermore, this elliptic function is real on the lines $\operatorname{Im}(t)=m \lambda \omega / 2$ and purely imaginary on the lines $R e(t)=m \omega / 2$. Consequently the zeros and poles of this function occur at the alternate points of intersection of this grid, and when we form the product $y(t) y(t+\omega / 2)$ they cancel, giving an entire function. Since this product is also doubly periodic, it must reduce to a constant equal to $y(-\omega / 4) y(\omega / 4)\left(=-k^{2} s a y\right)$.

Taking the first integral of the equation, we see that

$$
(\dot{y})^{2}=y^{4}+a_{1} y^{2}+c
$$

and since the equation is autonomous this holds for both $y(t)$ and $y(t+\omega / 2)$. Substituting $z=-k^{2} / y$, we obtain

$$
(\dot{z})^{2}=c k^{-4} z^{4}+a_{1} z^{2}+k^{4}
$$

showing that the constant of integration $c$ is equal to $k^{4}$. Hence the period $\omega$ is given by

$$
\int_{0}^{k} d y\left(y^{4}+a_{1} y^{2}+k^{4}\right)^{-1 / 2}=\omega / 4
$$

or

$$
\int_{0}^{1} d x\left(x^{4}+\alpha x^{2}+1\right)^{-1 / 2}=k \omega / 4
$$

where $\alpha=a_{1} k^{-2}$. Similarly, by considering $y(t) y(t+i \lambda \omega / 2)$, we obtain the result

$$
\int_{0}^{1} d x\left(x^{4}-\alpha x^{2}+1\right)^{-1 / 2}=k \lambda \omega / 4 .
$$

The existence of these integrals requires that $-2<\alpha<2$, and in the limiting cases we have

(1) for $\alpha \rightarrow 2 ; \lambda \rightarrow \infty, a_{1} \rightarrow 2 \pi^{2} / \omega^{2}$

(2) for $\alpha \rightarrow-2 ; \lambda \rightarrow 0, a_{1} \rightarrow-2 \pi^{2} / \lambda^{2} \omega^{2}$

It should also be noted that $a_{1}=0$ corresponds to $\lambda=1$, in which case $y$ can be represented in terms of the lemniscate sine.

It was shown in the earlier paper that the elliptic functions formed from doubly infinite sums of the basic functions

$$
g(t)=t^{-1}-(t+2 i \beta)^{-1}
$$

all satisfy $\ddot{y}=2 y^{3}+a y$ with $a>0$, where $a \rightarrow 2 \pi^{2} / \omega^{2}$ as the imaginary period tends to infinity. Hence for differential equations of the form $\ddot{y}=2 y^{3}+a y$, the monotonic solutions are only of the first type if $a \leqslant 0$, while if $a>0$ we have solutions of the first type if $(\dot{y})^{2}>a^{2} / 4$ when $y=0$ and of the second type if $(\dot{y})^{2}<a^{2} / 4$ when $y=0$; the separating solutions being $y= \pm \sqrt{(a / 2)} \cot (\sqrt{(a / 2)} t)$. 


\section{The Remaining SOLUTIONS}

The final class of solutions, which was overlooked in the previous paper, is obtained from the basic function

$$
g(t)=t^{-1}-(t+\alpha+i \beta)^{-1}
$$

with periods $\omega_{1}$ (real) and $\omega_{2}=2 i \beta$. The parameter $\alpha$ can take any real value, but no generality is lost in the double sums if we assume $0<\alpha<\omega_{1}$. The cases already discussed correspond to $\alpha=\omega_{1} / 2$ and $\alpha=0$ respectively.

The infinite sums over the periods give

$$
\Psi\left(t ; \omega_{1}\right)=\theta(\cot (\theta t)-\cot (\theta(t+\alpha+i \beta))), \theta=\pi / \omega_{1}
$$

and

$$
\Psi\left(t ; \omega_{2}\right)=\phi(\operatorname{coth}(\phi t)-\tanh (\phi(t+\alpha))), \phi=\pi / 2 \beta
$$

respectively. he first of these is not real on the real axis, while the second satisfies

$$
(\dot{y})^{2}=y^{4}-4 \phi \tanh (\phi \alpha) y^{3}+4 \phi^{2} y^{2}
$$

or

$$
\ddot{y}=2 y^{3}-6 \phi \tanh (\phi \alpha) y^{2}+4 \phi^{2} y .
$$

The doubly infinite sums lead to elliptic functions with poles and zeros arranged at the vertices of trapeziums. In the general case, however, these trapeziums are not congruent, and all that can be deduced is that the reciprocal of such a function is, to within scalar multiples and translations, another function of the same type.

\section{CONCLUSION}

The monotonic solutions for differential equations of the form $\ddot{y}=p(y)$, where $\dot{p}$ is a polynomial of degree 3 , which were reported in the earlier paper, satisfy a differential equation of the form

$$
(\dot{y})^{2}=y^{4}+a_{1} y^{2}+k^{4} .
$$

Because the solution functions are essentially self-reciprocal, it is possible to determine both the real and imaginary perioda from this relationship.

For the other monotonic solutions, no such simple relationship exists, although the class of solutions as a whole is closed under reciprocation.

\section{REFERENCES}

[1] A.S.Jones, 'Quasi-additivẹ solutions of non-linear differential equations', J. A ustral. Math. Soc. (Series A) 42 (1987), 92-116. 